\title{
Beyond multiregional and simple out-of-Africa models of human evolution
}

\author{
The past half century has seen a move from a multiregionalist view of human origins to widespread acceptance \\ that modern humans emerged in Africa. Here the authors argue that a simple out-of-Africa model is also outdated, \\ and that the current state of the evidence favours a structured African metapopulation model of human origins.
}

\section{Eleanor M. L. Scerri, Lounès Chikhi and Mark G. Thomas}

F or decades, polarized debates about human origins have swung between two major models. Classic multiregionalism viewed the majority of our ancestry as being spread across the Old World over the past one to two million years, and emphasized regional continuity. The recent and simple out-of-Africa (SOA) model proposed an expansion out of Africa in the past $100,000 \mathrm{yr}$ from a single region in Africa. Testing these models has undoubtedly improved our understanding of recent human origins, but with ever-richer archaeological, anthropological, genetic and palaeoecological data available, are they still useful? We argue that these formulations now constrain progress in human evolutionary studies and call for a shift to structured metapopulation models.

\section{A more realistic framework}

Genetic studies have repeatedly falsified classic multiregionalism. However, various aspects have returned under different guises, for example, refs. ${ }^{1,2}$, and thus it requires repeating: genetic data do not support an origin of Eurasian peoples primarily from locally evolving Homo populations over the past one to two million years, with a limited contribution from later African arrivals. Neither did humans 'leave' Africa; humans expanded their range, like other mammalian species and hominins before them. Recent findings estimating 1.5 to $2.8 \%$ genetic contribution from Neanderthals to non-African peoples and 0.3 to $5.6 \%$ contribution from Denisovans to East Asian and Oceanian peoples do not change this ${ }^{3}$. The remaining $\sim 91.8$ to $\sim 98.5 \%$ of the ancestry of people not living in Africa today still derives from Africa, probably in the past 100,000 yr (Fig. 1). Under a strict biological species concept, other large-brained nonAfrican hominins in that time period could be seen as variants of our own species. But origins imply ancestry, and our ancestry is primarily African. Fossils are unlikely to change that view.

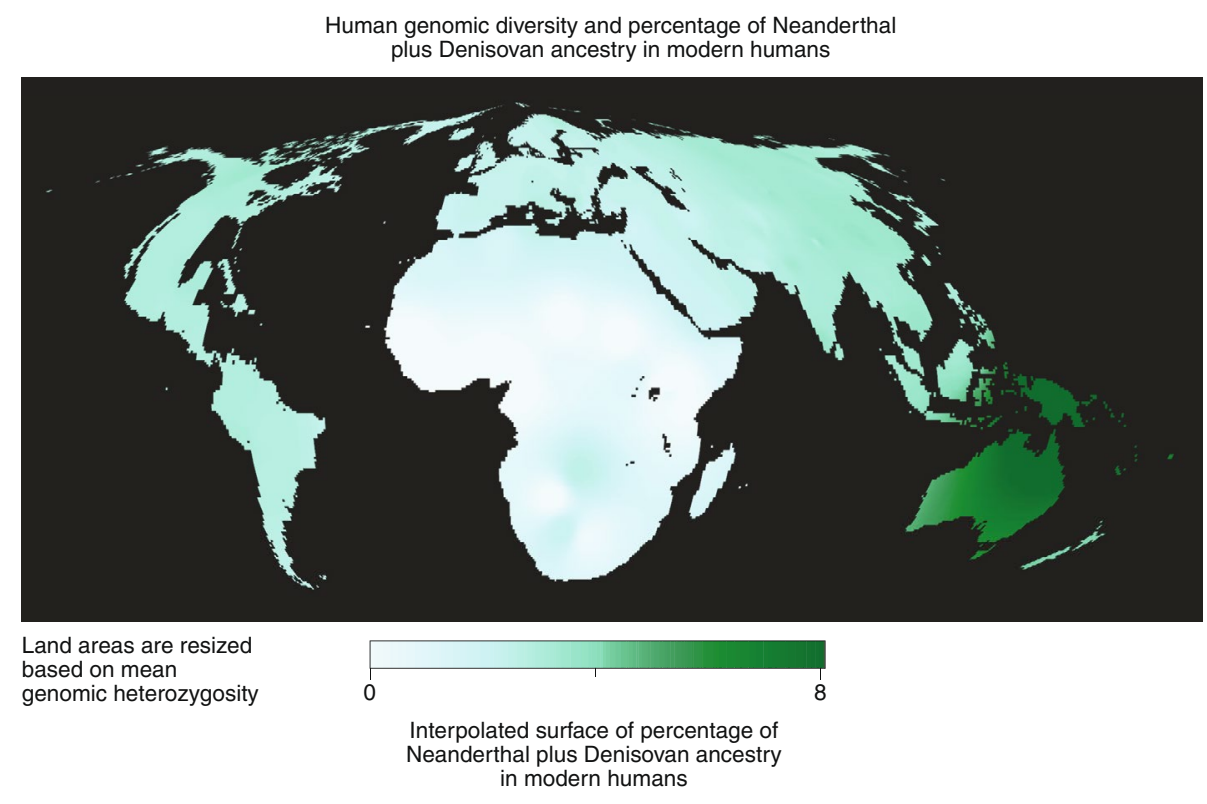

Fig. 1 | Cartogram with resized land area representing modern human genetic diversity and colour representing Neanderthal plus Denisovan ancestry. Mean regional genomic heterozygosity ${ }^{3}$ from 300 individuals was calculated for an adaptation of the United Nations geoscheme regional groups. These values provided the basis to distorting their respective land areas using a density equalizing approach ${ }^{22}$ to cartogram creation as implemented in the ScapeToad software package (version 1.1). The sample point locations were distorted based on the same deformation grid before interpolating the sum of the percentage Neanderthal plus Denisovan ancestry ${ }^{3}$ using a standard inverse distance-weighted approach. Finally the interpolated grid and cartogram were reprojected to the Mollweide projection. Credit: James Cheshire and Mark G. Thomas

The Homo sapiens lineage is widely thought to have separated from other Homo metapopulations - usually termed 'archaic hominins' - at least half a million years ago ${ }^{4}$. Notwithstanding the cladistic ambiguity of the term 'archaic hominins' - which is typically applied to both contemporaries and predecessors of H. sapiens - this is an unusually short time period for any neat split to occur in a longgeneration time taxon ${ }^{5,6}$. Contemporary humans are what remains of an ancient and complex variation in only one of its possible expressions. Palaeoanthropologists are aware of the problems of categorizing $H$. sapiens as a species ${ }^{4}$ as well as the need more generally to re-examine ambiguous terminology.

The widespread, although not universal, acceptance that modern humans admixed with Neanderthals and Denisovans has highlighted some of the problems of using simple evolutionary trees to represent human evolution, and the need to consider more reticulate models (that is, including gene flow between, or merging of branches), as some archaeologists, palaeoanthropologists and geneticists have argued ${ }^{7-12}$. While the concepts of 
trees, species and hybridization have had their utility, and may still be useful, they have become constraining and sometimes misleading in the emerging picture of human evolution.

At the same time, multiple lines of evidence indicate that Africa as a whole continent should be the focus of research if we want to understand human origins in a more realistic framework ${ }^{13}$. Fossil data show that the physical features characterizing contemporary humans did not appear progressively in one region. Instead, they appeared across Africa in a mosaic-like fashion, emerging at different times and in different combinations with diverse ancestral features, indicating a fragmented continental-wide trend towards the modern human form. Similarly, the Middle Stone Age - thought by many to reflect the emergence of modern cognition - appears to have polycentric origins across Africa. Palaeoclimate dynamics indicate corresponding fluctuations: habitable zones radically shifted throughout deep time and connections between them repeatedly appeared and disappeared.

These data indicate that early H. sapiens populations were strongly structured. In other words, they comprised sets of interconnected subpopulations forming a metapopulation (Fig. 2) that was distinct from other metapopulation sets corresponding to Neanderthals (for example, ref. ${ }^{14}$ ), Denisovans and possibly other hominin groups. A metapopulation model stresses the importance of structure and connectivity within evolutionary lineages as a continuous process of oscillating fission, fusion, gene flow and local extinction. Critically, it remains to be determined whether genomic data are better explained by such metapopulation models, or models assuming branching from a single ancestral population.

\section{Methodological implications}

A structured African metapopulation model, as in Fig. 2a, provides a highly flexible and generalizable framework for understanding human evolution. It can accommodate a wide range of previously hypothesized scenarios, including persistent or shifting isolation, 'archaic hominin' admixture, range expansion with regional persistence, and local extinction followed by recolonization from other subpopulations ${ }^{15}$.

While tree models can in theory be as complex as one wants by increasing the number of branches and by adding reticulation or hybridization, this is generally not how tree models have been used with genomic data. In most cases, tree topology is fixed a priori to a small a

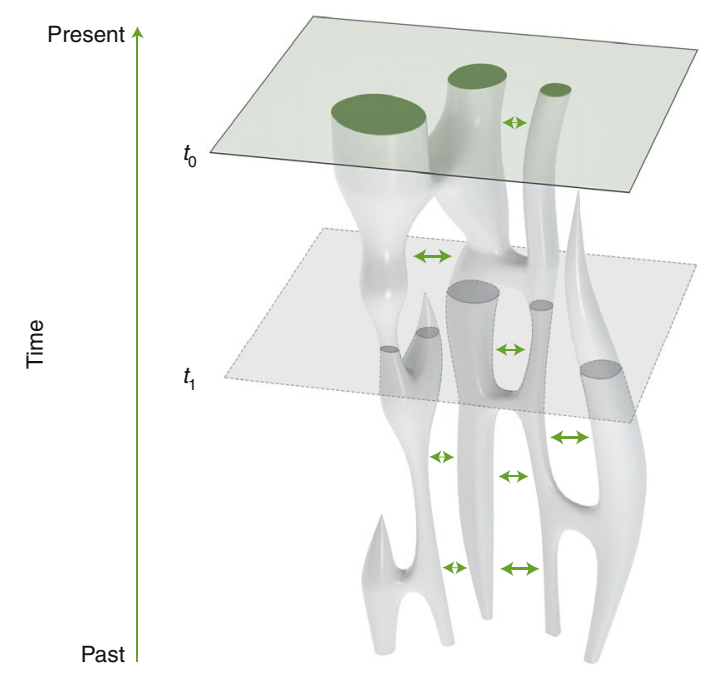

b

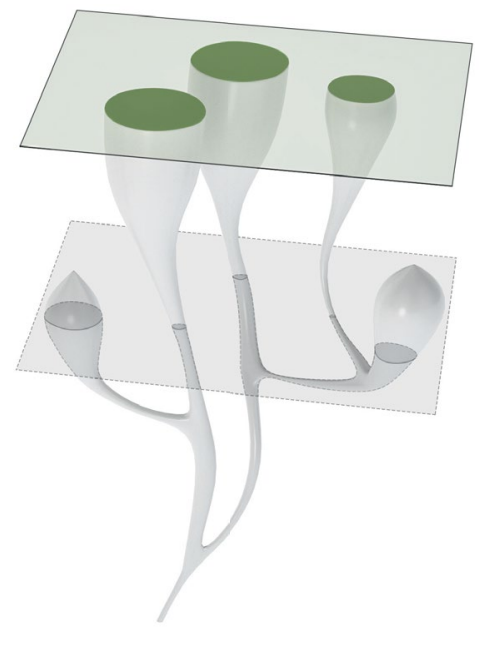

Fig. 2 | Different models of population history. $t_{0}$ and $t_{1}$ represent time slices in the present and past, respectively. a, A metapopulation, which includes population fission, fusion, gene flow and local extinction. b, A tree model, which includes population fission and extinction only. Inspired by figures from refs. 9,10 .

number of populations, often corresponding to whole continents or 'species' (for example, Neanderthals or Denisovans), which are represented as panmictic or homogenous branches. Admixture events are then invoked to explain genomic patterns that cannot be explained by the a priori-defined tree structure, and may thus be difficult to interpret ${ }^{7,16}$.

While such tree representations seem intuitive and are clearly useful when distant species are represented, they can be misleading when metapopulations are considered. Indeed, when key parameters of tree models such as splitting times of ancestral populations are inferred, it is unclear how they should be interpreted if reality is closer to a metapopulation model where no single ancestral population existed (Fig. 2). Another interpretation issue comes from the assumption of panmixia over large geographical areas. Population genetics theory shows that any shift in population structure will generate a spurious signal of population size change if panmixia is assumed ${ }^{11,13}$. In other words, a model ignoring population structure will infer and date events of population size changes that may have never occurred. Given that humans would never have been panmictic at anything other than the very local scale, it is unclear how estimating such size changes would increase our understanding of human evolution ${ }^{12,14}$.

Metapopulation models have a rich theoretical history ${ }^{17}$, although their use in demographic inference has been limited.
While it may be challenging to explore highly parameterized metapopulation models that detail individual isolation and mixing events, the advantage of this family of models is that they can represent rather complex demographies without necessarily requiring a very large number of parameters. This can be achieved by stressing long-term population dynamics in relation to shifting isolation and gene flow (Fig. 2), rather than focusing on the dating of splitting events. We thus argue that effort should be concentrated on metapopulation models, starting with simple ones, to identify key features of long-term population history (for example, ref. ${ }^{14}$ ).

\section{Theoretical implications}

In this general framework, the SOA model should be rejected in favour of one including dynamic connections and disconnections between geographically structured subpopulations as a consequence of ecological changes. Such processes shape patterns of genetic, morphological and cultural variation and have the potential to better explain archaeological, palaeoanthropological and genetic data, while being more consistent with what is known of Pleistocene climate oscillations, than either of the outdated models.

A generalized concept of structure therefore also offers more theoretical flexibility to describe and explain patterns of human evolution. For example, structured models better explain the evolution of various derived morphological features 
associated with modern humans, such as a globular braincase, a small and gracile face, and $\operatorname{chin}^{4,9}$. These features first appear separately at different times in different fossils across Africa, but are only found together in the past 100 to $40,000 \mathrm{yr}$. A view whereby these and other derived features evolve separately in distinct subpopulations, and spread asynchronously through fluctuating gene flow, would explain the lack of sequentiality in their appearance and lessen the temptation to assign subspecies labels to different fossils.

Structured models may also help to explain both the more widespread generic features of Middle Stone Age material culture (for example, denticulates) and the fluctuating appearance of region-specific technologies (for example, tanged tools) ${ }^{9}$. Several theoretical studies show that larger populations maintain both greater cultural complexity ${ }^{18}$ and more genetic diversity. However, population structure results in the maintenance of more genetic diversity at the metapopulation level as an inverse function of connectivity, but it also leads to lower local genetic diversity and reduced cultural complexity at both the local and metapopulation levels ${ }^{19}$. Given the similar effects of structure on local complexity, but opposing effects on overall genetic and cultural complexity, it is possible that appropriate forms of structured models are the only means of jointly explaining genetic and archaeological patterns.

Finally, a structured African metapopulation model is naturally spatiotemporally explicit (Fig. 2), and so can be integrated with knowledge of shifting palaeoclimates and palaeoecologies. For example, the role that contiguous environmental zones across the SaharoArabian arid belt played in both limiting, and at times facilitating, gene flow can be explored without limiting researchers to invoking discrete splitting events between isolated populations.

In sum, a structured African metapopulation model moves beyond old classic multiregionalism versus SOA debates, along with the schemes and terminologies that support them. While for the sake of convenience such pan-African origins have been dubbed 'African multiregionalism ${ }^{15,20}$, this should not be confused with classic multiregionalism or a soft version of it. Classic multiregionalism is about geographical continuity, and variations of it have been linked to beliefs that there are real, autochthonous people whose ancestors have 'always been here'. In contrast, the SOA model is problematic in its invocation of a single 'colonizing' population from which we all descend. Both underplay what makes most mammalian species successful: their ability to expand their ranges, exploit new environments, adapt to changing environments and periodically maintain connectivity between populations. Like any other invasive species, humans have repeatedly expanded both within and beyond their African tropical comfort zone ${ }^{21}$.

Understanding humans and their evolution will require models of shifting population structure where complete isolation is seen as the exception, and not the rule. This does not imply that shifting structure was the only process impacting human diversity or that structure only began to play a role at a certain point in human evolution. Indeed, it seems likely that complex models will be required to explain the ever-richer fossil record of highly divergent hominins apparently living alongside $H$. sapiens across the Old World. Structured metapopulation models offer a way to acknowledge the palaeontological, archaeological and genetic evidence for a recent African origin with limited gene flow from non-African metapopulations, without falling into overly polemic and restrictive debates. Any model that would claim to represent human evolution would have to satisfactorily explain patterns of variation in genetic, morphological and cultural data components, and be consistent with the climatic changes that have shaped our ecologies during the Pleistocene. This is probably one of the greatest and most exciting challenges of the next decade of human evolution research.

\footnotetext{
Eleanor M. L. Scerri (D) 1*, Lounès Chikhi' ${ }^{2,3}$ and Mark G. Thomas (D) 4,5

${ }^{1}$ Pan African Evolution Research Group, Max Planck Institute for the Science of Human History, Jena, Germany. ${ }^{2}$ Laboratoire Évolution et Diversité Biologique (EDB UMR 5174), Université de Toulouse Midi-Pyrénées, CNRS, IRD, UPS, Toulouse, France. ${ }^{3}$ Instituto Gulbenkian de Ciência, Oeiras, Portugal. ${ }^{4}$ Research Department of Genetics, Evolution and Environment, University College London,
}

London, UK. ${ }^{5}$ UCL Genetics Institute, University College London, London, UK.

*e-mail:scerri@shh.mpg.de

Published online: 23 September 2019

https://doi.org/10.1038/s41559-019-0992-1

References

1. Athreya, S. \& Wu, X. Am. J. Phys. Anthropol. 164, 679-701 (2017)

2. Derevianko, A. P. Archaeol. Ethnol. Anthropol. Eurasia 39, 2-31 (2011)

3. Mallick, S. et al. Nature 538, 201-206 (2016).

4. Stringer, C. Phil. Trans. R. Soc. Lond. B 371, 2015.0237 (2016).

5. Holliday, T. W. in Vertebr Paleobiol Pa Vertebrate Paleobiology and Paleoanthropology (eds Hublin, J.-J., Harvati, K. \& Harrison, T.) 281-297 (Springer Netherlands, 2006).

6. Mallet, J. Phil. Trans. R. Soc. Lond. B 363, 2971-2986 (2008).

7. Eriksson, A. \& Manica, A. Proc. Natl Acad. Sci. USA 109 13956-13960 (2012).

8. Ackermann, R. R., Mackay, A. \& Arnold, M. L. Evol. Biol. 43, 1-11 (2016).

9. Harding, R. M. \& McVean, G. Curr. Opin. Genet. Dev. 14, 667-674 (2004).

10. Lahr, M. M. \& Foley, R. A. Am. J. Phys. Anthropol. 107, 137-176 (1998)

11. Marjoram, P. \& Donnelly, P. Genetics 136, 673-683 (1994).

12. Goldstein, D. B. \& Chikhi, L. Annu. Rev. Genomics Hum. Genet. 3 , 129-152 (2002)

13. Scerri, E. M. L. et al. Trends Ecol. Evol. 33, 582-594 (2018).

14. Rodriguez, W. et al. Heredity 121, 663-678 (2018).

15. Henn, B. M., Steele, T. E. \& Weaver, T. D. Curr. Opin. Genet. Dev. 53, 148-156 (2018).

16. Kuhlwilm, M. et al. Nature 530, 429-433 (2016).

17. Hanski, I. \& Gaggiotti, O. in Ecology, Genetics and Evolution of Metapopulations (eds Hanski, I. \& Gaggiotti, O. E.) 3-22 (Academic Press, 2004).

18. Powell, A., Shennan, S. \& Thomas, M. G. Science 324, 1298-1301 (2009)

19. Henrich, J. et al. Proc. Natl Acad. Sci. USA 113, E6724-E6725 (2016).

20. Klein, R. G. Evol. Anthropol. 28, 179-188 (2019).

21. Harvati, K. et al. Nature 571, 500-504 (2019).

22. Gastner, M. T. \& Newman, M. E. Proc. Natl Acad. Sci. USA 101, 7499-7504 (2004)

Acknowledgements

We thank J. Cheshire for generating Fig. 1, and M. O'Reilly and C. Pantiru for Fig. 2 design. We also thank C. Stringer and $\mathrm{H}$. Groucutt for comments on the manuscript. E.M.L.S.'s work was supported by a Marie Skłodowska Curie Actions Fellowship and the Max Planck Society. M.G.T. is supported by a Wellcome Trust Senior Research Fellowship (grant 100719/Z/12/Z, 'Human adaptation to changing diet and infectious disease loads, from the origins of agriculture to the present'). L.C. is supported by the French Laboratory of Excellence project 'TULIP' of the Agence Nationale de la Recherche (ANR-10-LABX-41; ANR-11-IDEX-0002-02), the LIA BEEG-B (Laboratoire International Associé-Bioinformatics, Ecology, Evolution, Genomics and Behaviour) between the CNRS and Instituto Gulbenkian de Ciência, the ANR Investissement d'Avenir grant (CEBA: ANR-10-LABX-25-01) and the FCT (Fundação para a Ciência e a Tecnologia) through the INFRAGECO and DISPO projects (Biodiversa/0003/2015 and PTDC-BIA-EVL/30815/2017).

Competing interests

The authors declare no competing interests. 